\title{
A study on the prevalence Of Depression in caregivers of patients with Alcohol Dependence and its influence on caregiver help seeking tendency
}

\author{
Sushith Sugathan Chennatte ${ }^{1}$, Jenny Joseph ${ }^{2}$, Arun Padmanandan ${ }^{3}$ \\ ${ }^{1}$ Assistant Professor, Department of Psychiatry, PSG Institute of Medical Science and Research, \\ Coimbatore, Tamil Nadu. \\ ${ }^{2}$ MBBS[Year 3], PSG Institute of Medical Science and Research, Coimbatore, Tamil Nadu. \\ ${ }^{3}$ Assistant Professor, Department of Community Medicine, PSG Institute of Medical Science and \\ Research, Coimbatore, Tamil Nadu
}

Corresponding author: Sushith Sugathan Chennatte

Email -dr.sushiths@yahoo.com

\begin{abstract}
Introduction: Caregivers of patients with alcohol dependence often refrain from asking for help for deaddiction. This leads to worsening of the condition due to delay of planned interventions for treatment of dependence and further adds to the care giver burden..

Methodology: In this cross-sectional study we used consecutive sampling of patients with alcohol dependence syndrome who got admitted into the gastroenterology in-patient ward. Their corresponding caregivers included for the study were spouse/parent/sibling. The prevalence of depressive symptoms was screened using the Becks depression inventory [BDI]. We assessed the likelihood of help seeking in the caregiver for their affected relative's addiction problem by survey questionnaire formulated in Tamil. To compare the BDI scores between the help seeking and non-help seeking caregivers, we used the one way ANOVA test.

Results: In the whole sample, 34 [out of 39] caregivers [87.18\%] had depressive symptoms score of above 17 [clinically significant depression] on the BDI depression scale. The mean BDI score was significantly higher in those caregivers who did not seek help for their relative's addiction and this was statistically significant [ $p$ value $=0.04$ ]

Conclusion: The identification of depressive symptoms in the caregiver is important in addressing care giver burden and thus in improving psychosocial support for persons with alcohol dependence.
\end{abstract}

Keywords: Care Giver Depression, Help seeking, Alcohol dependence

[Paper received $-20^{\text {th }}$ June 2020, Peer review completed $-29^{\text {th }}$ July 2020, Accepted $-30^{\text {th }}$ July 2020]

\section{INTRODUCTION}

Family plays a central role in providing care to the alcohol dependent patients especially in an Indian setting where inter-dependence is prominent. An alcohol dependent person creates imbalance in all aspects of family life and causes enormous burden to the caregivers [Fig.1]. In most cases the spouse/parent of the patient is the most affected and experience severe emotional instability.

According to the Global Status Report on Alcohol, Alcohol Use Disorders account for $1.4 \%$ of the worldwide burden of diseases [1]. A nationwide Indian study on alcohol and drug abuse by Sarkar et al estimated the prevalence of alcohol use as $21.4 \%$ [2]. Alcohol dependence affects not only the patient but also their caregivers. It causes physical and emotional distress and financial burden due to addictive behaviour of the dependent individual. This has a serious impact on the lives of the caregivers [3].

The role of the caregiver in helping their family member with alcohol dependence includes financial assistance, management of illness symptoms and also the engagement and retention of the patient in 
treatment [4]. This requires a lot of resilience and motivation on part of the caregiver in providing such exhaustive psychosocial support for their family member with alcohol dependence especially when there are frequent relapses. Research also indicates that care giving itself can be an independent risk factor for mortality [5].

If caregivers refrain from asking for help it would be keep adding to their burden due to worsening of the condition due to delay of planned interventions for treatment of dependence. A study on the impact of partner alcohol problems on women's physical and mental health was carried out by Dawson et al. [6]. It was found that these women had increased incidences of mood disorders and depressive episodes than women whose partners did not have alcohol problems. Tempier et al had studied the consequences of alcoholism on the mental health of the spouses and found that there was a high level of psychological distress among the wives of male lifetime at-risk drinkers [7].

Studies show that emotional burdens related to care of alcohol dependent patients warrant attention from health care and mental health care professionals [8]. On literature review we were able to identify Indian studies looking at caregiver burden in patients with alcohol dependence [9-11], but there was paucity of studies analysing the correlation of depressive symptoms in care givers and help seeking for their relatives with alcohol dependence patients per se.

Figure1. Figure depicting effect of non-help seeking tendency on caregiver burden

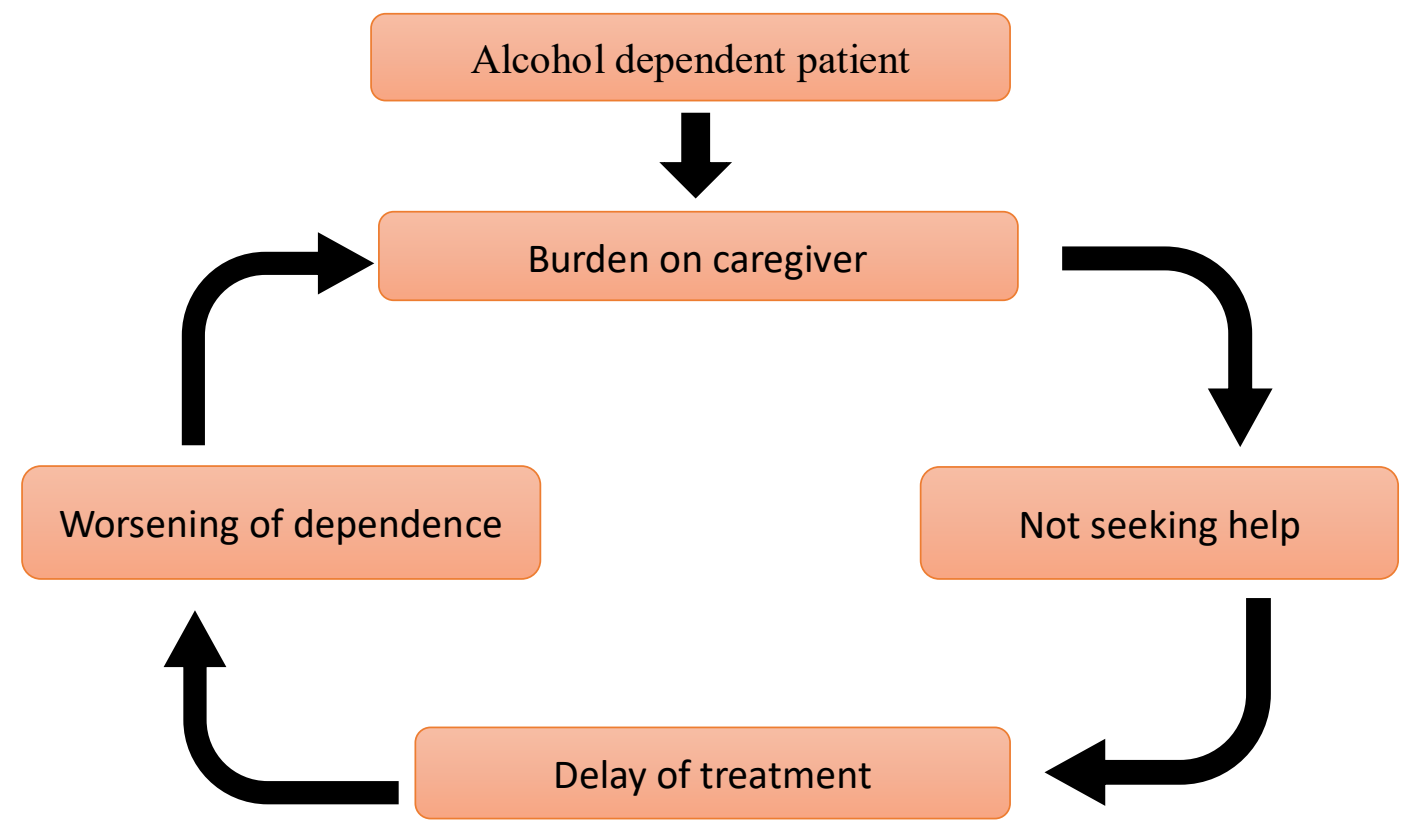

Objective of the study:

1] To study prevalence of depressive symptoms in the caregivers of alcohol dependent patients who were admitted as in-patient for gastroenterological problems.

2] To compare the mean becks depression inventory [BDI] scores between help-seeking carers and non-help seeking carers. Help seeking was defined in the sense of seeking deaddiction for their relative.

\section{METHODOLOGY}

This was a cross-sectional study in which we used consecutive sampling of patients getting admitted into the in-patient gastroenterology ward at a private multi-specialty hospital in the district of Coimbatore at Tamil Nadu State in India. The study had been approved by the institute's Human Ethics Committee [Project No. 18/093]. After getting informed consent we collected data on socio demographic and clinical profile of the 
patients and their corresponding primary caregivers. This was done by the author [JJ] using a semi structured data collection profoma [Figure 2]. We set the sample size of 80 caregivers, similar to a previous study which had analyzed the likelihood of help seeking in caregivers of patients with substance use [16].

Inclusion criteria for the relation of caregiver were that they should have been either the spouse/parent/sibling to the patient. The corresponding patient should have satisfied the criteria for alcohol dependence syndrome confirmed by a psychiatrist by administering SCID for DSM 5. All alcohol dependent patients admitted to the gastroenterology ward were seen by a consultation-liaison psychiatrist as per department policy. The age of caregiver must be over 18 years. The patient and caregiver should have given informed consent for the study.

If the patient being cared for by the caregiver had co-morbid physical or psychiatric disorders which were not associated to alcohol use, then those caregivers were excluded from the study. Care givers with past history of psychiatric illness or co-morbid medical illness were excluded from the study.

The consultation liaison psychiatrist assessing patients in the gastroenterology ward utilized the SCID interview for Diagnostic and Statistical Manual of Mental Disorders, Fifth Edition [DSM-5] for assessing alcohol dependence syndrome in the patient [12]. The Beck's Depression Inventory [validated Tamil translated version] was used for assessing depressive symptoms in the caregiver. This was a self-rating depression scale that was completed by the caregivers themselves after being explained by the author [JJ]. $\mathrm{BDI}$ is a 21-item; self-report rating inventory that measures characteristic attitudes and symptoms of depression. It has high internal consistency, with alpha coefficients of 0.86 and 0.81 for psychiatric and nonpsychiatric populations respectively [13-14]. For the patients corresponding to these caregivers, author 'JJ' had administered the "Severity of Alcohol dependence questionnaire" [validated Tamil translated version] for assessing severity of alcohol dependence [15].

We assessed the likelihood of help seeking in the caregiver for their affected relative's addiction problem by asking them 3 questions with yes or no reply options framed in the local language Tamil. The screening questions were posed in the order such as: a] Do you know whether your relative has a problem with alcohol addiction? b] Are you likely to ask for help for your relative's addiction problem? We classified the caregivers as help seeking if they answered yes to both the questions and as non-help seekers if they had answered yes to question ' $a$ ' but no to question ' $b$ '.

\section{Statistical Analysis}

We used the SPSS software version 19 for Windows for statistical analysis. Case and control matching in our sample was done to randomly match only for socio demographic variables like age, gender and socioeconomic and demographic status and clinical variable of dependence severity in the patient using the SADQ score. This was done by comparing the mean differences and associated $\mathrm{p}$ value of these variables between the 2 groups [those willing and those not willing to seek help].

We used descriptive statistics to calculate the mean and the standard deviation. Among the help seeking and non-help seeking groups, we used descriptive statistics to get the mean and standard deviation of continuous variables. Among the help seeking and non-help seeking groups, we used chi-square test to compare dichotomous variables like gender, education [secondary level and below secondary levels], socioeconomic status [lower and middle], relation of caregiver[spouse], presence of psychological symptoms in the relative, presence of social and occupational dysfunction in the relative, presence of past deaddiction treatment, presence of co-morbid substance abuse. Student $t$ test was used to compare continuous variables like age, SADQ total scores, duration of alcohol intake. To compare the mean Becks depression inventory [BDI] scores between the help seeking and non-help seeking carers, we used one way ANOVA test.

\section{RESULTS}

\section{Sociodemographic Variables}

Among the total sample of 39 care givers, they were divided into two groups such as the help seeking [N= $21,53.85 \%]$ and the non-help seeking group $[\mathrm{N}=18,46.15 \%]$, based on their responses in the help-seeking questionnaire. 
There was no significant difference among variable of socioeconomic status between the help seeking and non-help seeking group. The care givers were predominantly female gender in both groups and there was no significant difference between the mean ages of caregivers in the help seeking and non-help seeking groups. Regarding socioeconomic status, $50 \%$ in both the groups were from lower socio economic strata. Regarding relation of the caregivers to the patient, more than $50 \%$ of caregivers were the wives of the patients in both the groups. Mothers constituted around 14 and $22 \%$ of the caregivers in the help seeking and non-help seeking groups respectively. Remaining percentage [ $28 \%$ in help seeking and $22 \%$ in non-help seeking] of the caregivers constituted either brother, child or father of the patient. No significant difference was found in the relation of caregivers between the two groups. [Table1]

Figure 2: Methodology

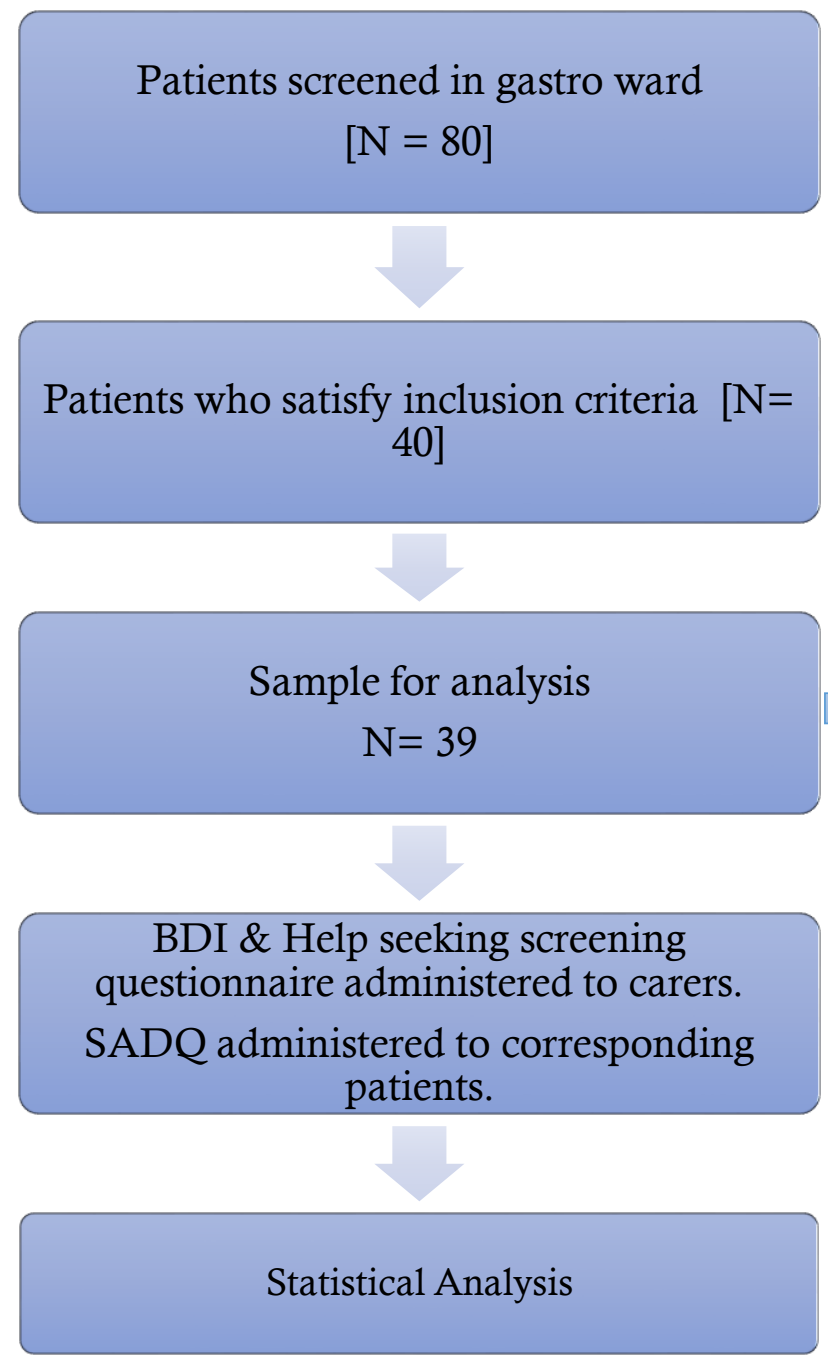

\section{Clinical Variables}

Clinical variables influencing severity of the alcohol dependence such as duration of alcohol intake, mean SADQ score, comorbid substance abuse and family history of alcohol dependence did not differ significantly between the two groups. But there were some significant differences in terms of certain clinical variables which may have influenced the results and this has been looked at under the discussion part of the paper. [Table 2] 
Table 1: Comparison of Socio-demographic Variables

\begin{tabular}{|c|c|c|c|}
\hline $\mathbf{N}[\%]$ & $\begin{array}{c}\text { Help seeking care } \\
\text { giver } \\
{[\mathbf{N = 2 1 ]}}\end{array}$ & $\begin{array}{c}\text { Non help seeking } \\
\text { care giver } \\
{[\mathbf{N = 1 8}}\end{array}$ & $\begin{array}{c}\text { Significance } \\
\text { P value }\end{array}$ \\
\hline Gender [Female] & $15[71.43 \%]$ & $15[83.33 \%]$ & 0.38 \\
\hline Mean Age in years & 43.43 & 47.11 & 0.20 \\
\hline $\begin{array}{c}\text { Lower } \\
\text { Socioeconomic status }\end{array}$ & $12[57.14 \%]$ & $9[50 \%]$ & 0.46 \\
\hline Secondary Education & $12[57.14 \%]$ & $9[50 \%]$ & 0.46 \\
\hline $\begin{array}{c}\text { Relation of caregiver } \\
\text { to patient [Spouse] }\end{array}$ & $12[57.14 \%]$ & $10[55.56 \%]$ & 0.39 \\
\hline $\begin{array}{c}\text { Relation of caregiver } \\
\text { to patient [Mother] }\end{array}$ & $3[14.28 \%]$ & $4[22.22 \%]$ & 0.52 \\
\hline
\end{tabular}

Table 2: Comparison of clinical variables

\begin{tabular}{|l|c|c|c|}
\hline \multicolumn{1}{|c|}{ N [\%] } & $\begin{array}{c}\text { Help seeking care } \\
\text { giver } \\
{[\mathbf{N = 2 1 ]}}\end{array}$ & $\begin{array}{c}\text { Non help } \\
\text { seeking care } \\
\text { giver } \\
{[\mathbf{N}=18]}\end{array}$ & $\begin{array}{c}\text { Significance } \\
\text { P value } \\
{\left[^{*}<\mathbf{0 . 0 5}\right]}\end{array}$ \\
\hline Mean duration of alcohol intake & 13.29 & 13.22 & 0.49 \\
\hline Mean SADQ score & 27.24 & 27.83 & 0.41 \\
\hline $\begin{array}{l}\text { No. of patients with Psychological } \\
\text { symptoms of dependence }\end{array}$ & $18[85.71 \%]$ & $14[77.78 \%]$ & 0.51 \\
\hline $\begin{array}{l}\text { No. of patients with Social } \\
\text { dysfunction due to dependence }\end{array}$ & $10[47.62 \%]$ & $3[16.67 \%]$ & $\mathbf{0 . 0 4}^{*}$ \\
\hline $\begin{array}{l}\text { No. of patients with Occupational } \\
\text { dysfunction due to dependence }\end{array}$ & $12[57.14 \%]$ & $3[16.67 \%]$ & $\mathbf{0 . 0 1}$ \\
\hline $\begin{array}{l}\text { No. of patients with Co-morbid } \\
\text { substance abuse }\end{array}$ & $13[61.9 \%]$ & $10[55.56 \%]$ & 0.69 \\
\hline $\begin{array}{l}\text { No. of patients with Family history } \\
\text { of alcohol dependence }\end{array}$ & $9[42.86 \%]$ & $9[50 \%]$ & 0.66 \\
\hline $\begin{array}{l}\text { No. of patients with Past de- } \\
\text { addiction treatment history }\end{array}$ & $7[33.33 \%]$ & $13[72.22 \%]$ & $\mathbf{0 . 0 2}$ \\
\hline
\end{tabular}

Primary outcome measure: Prevalence of depression in care giver

In the whole sample, 34 out of 39 caregivers [87.18\%] had depressive symptoms score of above 17 [clinically significant depression] on BDI scale. Out of this 34 care givers with clinically significant depression, 9 caregivers [26.47\%] had borderline clinical severity on BDI, 14 [41.18\%] had moderate severity depression was, 9 [26.47\%] had severe depression and 2 [5.88\%] had extremely severe depression on BDI rating [Figure 3]

\section{Secondary Outcome measure: Comparison of mean BDI scores}

On comparison of the mean BDI scores between the help-seeking and non-help-seeking caregiver groups, the non-help seeking group had higher mean BDI score [66.36] than the help-seeking group [58.18] and this difference was statistically significant $[p=0.005]$. It was also important to note that both groups of care givers had reported mean score indicating more than severe depression [more than 40] on BDI rating. 
Figure 3 Distribution of depression severity [as per BDI scores] in the care givers

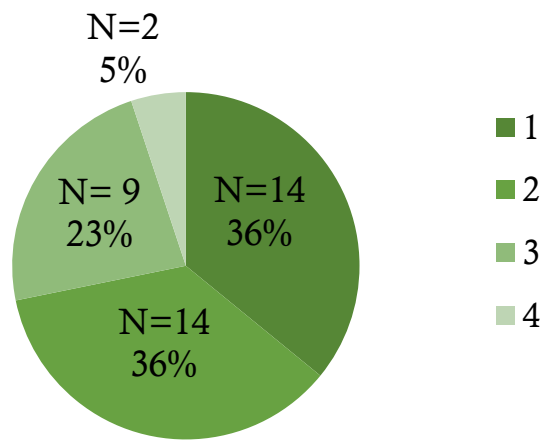

BDI scores ${ }^{*}$

$17-20$

[Borderline clinical depression]

21-30 [Moderate depression]

31-40 [Severe depression]

$>40$ [Extreme depression]

*1[Borderline clinical depression], 2[Moderate depression], 3[Severe depression], 4[Extreme depression].

Table 3 - Comparison of mean BDI scores between help seeker and non-help seeker

\begin{tabular}{|c|c|c|c|}
\hline & $\begin{array}{c}\text { Number } \\
\text { [Total N= 39] }\end{array}$ & $\begin{array}{c}\text { Mean BDI score } \\
\text { [Depression severity] }\end{array}$ & $\begin{array}{c}\text { P value } \\
\text { [Student T test for } \\
\text { comparison of } \\
\text { mean BDI scores] }\end{array}$ \\
\hline $\begin{array}{c}\text { Help seeking } \\
\text { Caregiver }\end{array}$ & $\begin{array}{c}21 \\
{[53.85 \%]}\end{array}$ & 58.18 & \multirow{2}{*}{$\mathbf{0 . 0 0 5}^{*}$} \\
\cline { 1 - 3 } $\begin{array}{c}\text { Non Help seeking } \\
\text { Caregiver }\end{array}$ & $\begin{array}{c}18 \\
{[46.15 \%]}\end{array}$ & 66.36 & \\
\hline
\end{tabular}

\section{DISCUSSION}

There have been many studies on care giver burden in alcohol dependence. A study in Chandigarh had assessed care giver burden in 120 subjects with alcohol and/or opioid dependence, and found around 95$100 \%$ reported severe care giver burden on the FBIS scale [18]. Further, there was a study done at Chennai on 200 people with alcohol dependence and their caregivers. It had shown that the severity of alcohol dependence was positively correlated [coefficient of 0.67] with severity of care giver burden. Thus more the severity of dependence more is the caregiver burden [19]. A national representative study in French adult population of 1018 participants in 2013 had looked further into burden of caregiver and found it being significantly influenced by social, behavioural and physical complications of alcohol dependence [20].

Thus, we can see that the caregiver burden is well established in alcohol dependent population. In our study we went further to analyse the prevalence of depression in the caregivers of alcohol dependent patients in our sample and to compare severity of depression in help seeking and non-help seeking groups of care givers. This would be a first step in looking at the significance of depression severity in help seeking tendency of caregivers in our sample burdened with alcohol dependence.

Out of 39 caregivers, $46.15 \%$ [ $N=21]$ of care givers were found to be unlikely to seek help for their relative with alcohol dependence and they formed the "non-help seeking group" and the remaining formed the "help-seeking group" [53.85\%, $\mathrm{N}=18]$ in our study. This was based on their responses in the questionnaire. Our finding was similar to a study done in Cleveland, Ohio on caregivers of 82 women with alcohol dependence. This latter study had shown that though caregivers undergo moderate levels of caregiver burden, only about $45 \%$ of caregivers were unlikely to ask for help. 
The two groups that is the help seeking and the non-help seeking caregivers did not differ significantly in terms of socio demographic profile. Most common among the type of caregivers were the spouses of the patients, followed by mothers of the patients.

Now let us discuss regarding the clinical profile of the patients for whom the caregivers were responsible for. We already know that physical complications in alcohol dependence can worsen the caregiver burden. All the patients in our sample were those who had been admitted in the ward for alcohol related gastro intestinal problems. Thus we chose a homogenous sample of alcohol dependent patients from a gastroenterology inpatient setup so that there is no significant difference between the help seeking and non-help seeking care givers in this aspect. Regarding the medical profile of the patients admitted in the gastro ward, 32 patients [82\%] had alcohol related liver disease and remaining 7 patients [18\%] had alcohol related pancreatic disease. All patients had been already medically stabilized at the time of interview in the ward which was part of our inclusion criteria. There was also no significant difference between the mean duration of alcohol intake or severity of alcohol dependence [as measured on SAD Q scale] between the help seeking and nonhelp seeking carer groups.

Further, we analysed the depressive symptomatology in the whole sample of caregivers. We found that 87 $\%$ of the whole sample had clinically significant depression [BDI scores of above 17]. When we further compared the severity of depression between the help seeking and the non-help seeking caregivers, we found that the mean BDI score was significantly higher in the non-help seeking group. This was an important finding as the risk of worsening of burden in the non-help seeking group could be further complicated by higher depression severity in that group. Furthermore it is clinically important to note that the mean BDI score in either of the groups were falling between the extremely severe depression range on BDI rating [over 40]. This shows the disabling capability of the depression in this cohort of caregivers and the compounding effect that it could have if the care giver is not seeking help.

When we compared the clinical profile between the two groups, we had some more significant findings that could also be correlated to help-seeking tendency in the care givers. We found a higher number of patients with social and occupational dysfunction [as assessed in SCID interview] to be cared for in the help-seeking care giver group. Care givers in the help seeking group had higher proportion [47.6\%] of socially dysfunctional alcohol dependent relatives compared to non-help seeking group [16.7\%] and this was statistically significant $[p=0.04]$. Similarly, the proportion of occupationally dysfunctional alcohol dependent relatives were higher in the help-seeking group [57.1\%] as compared to the non-help seeking group [16.7\%] and this difference was also statistically significant $[p=0.01]$.

Still another significant finding was that the non-help seeking group had higher proportion of relatives [72.2\%] who have had history of deaddiction treatment in the past. This was statistically significant with a $p$ value of 0.02 . Thus the experience of past deaddiction treatment may have influenced the current non-help seeking tendency in this group. But a limitation of our study was that we hadn't qualitatively assessed the nature of their past experience with deaddiction treatment to understand this further.

The high mean severity of depression in our care giver sample could also be explained by the profile of the patients for whom the care givers were responsible for. The severity of alcohol dependence and also the physical complications for which patient had been admitted for in the gastro ward and also the social and occupational dysfunction secondary to alcohol dependence could have been contributing to the depressive symptomatology. Also we had ruled out history of psychological illnesses in the profile of the caregivers in the sample. But the limitation of our study was that we hadn't looked into the qualitative report of the caregiver's specific concern regarding their alcohol dependent relative and we hadn't thus confirmed whether that was the actual contributing factor to their depression.

A limitation of this study was that our study objective was analysed using just a "yes" or "no" survey whereas a better method would have been to use a standardized scale to assess the likelihood of help seeking in caregiver. Though a recent scoping review of help seeking measures had identified 10 validated scales for measuring help seeking, these were scales directed towards the patient's help seeking behaviour rather than that of care givers. [17] Further to say, these scales were not specific to alcohol dependence and were covering mostly disorders such as schizophrenia, depression, anxiety and ADHD. Thus due to nonavailability of such a standardized and specific scale so far for caregivers of alcohol dependent patients, we decided to initially conduct a survey of help seeking behaviour and then in future studies to further analyse 
these behaviours using a standardized scale specific to the findings that emerge from our current study. In a previous study with a similar objective they had also mentioned a similar limitation of non-availability of a standardized scale specific to caregiver of alcohol dependent patients [16]. In their study too they had used just a single question survey on caregiver's likelihood of help seeking and had rated it on a 4-point likert scale.

Despite small sample size of this study, we were able to get significant findings when comparing the help seeking and non-help seeking care giver groups. The results have important clinical implications in reducing the burden of the caregivers of patients with alcohol dependence by identifying depressive symptoms in the care givers. The results of the study could help in understanding the role of depressive symptoms in the caregiver as an important prognostic factor in the help seeking tendency. Early interventions in addressing the depressive symptoms in the caregiver should be an important part of the management of alcohol dependent patient.

\section{CONCLUSION}

Factors such as high severity of depression in the caregiver, and past history of failed multiple deaddiction treatments in the past could negatively influence the help seeking tendency in care giver. Whereas, social and occupational dysfunction in the alcohol dependent patient maybe driving force for help-seeking tendency in the caregiver. Future studies are warranted to look into the caregiver's qualitative reports of reasons attributable to their depression. Qualitative studies are also warranted to analyse the subjective perceptions of past deaddiction treatment experiences that may have influenced the care giver's current help seeking tendency for their relative.

\section{REFERENCES}

1. World Health Organization, Global Status Report on Alcohol, World Health Organization, Geneva, Switzerland, 2004.

2. Sarkar AP, Sen S, Mondal S, Singh OP, Chakraborty A, Swaika B. A study on socio-demographic characteristics of alcoholics attending the de-addiction center at Burdwan medical college and hospital in West Bengal. Indian j Pub Health 2013;57(1):33-35.

3. Kaur D, Ajinkya S. Psychological impact of adult alcoholism on spouses and children. Med J DY Patil Univ 2014;7:124-7.

4. Provencher HL, Perreault M, St-Onge M, Rousseau M. Predictors of psychological distress in family caregivers of persons with psychiatric disabilities. J Psychiatr Ment Health Nurs 2014;10(5):592-607.

5. Schulz R, Beach SR. Caregiving as a risk factor for mortality: The Caregiver Health Effects Study. JAMA 1999;282(23):2215-19.

6. Dawson DA, Grant BF, Chou SP, Stinson FS: The impact of partner alcohol problems on women's physical and mental health. J Stud Alcohol Drugs 2007;68(1):66-75.

7. Tempier R, Boyer R, Lambert J, Mosier K, Duncan CR. Psychological distress among female spouses of male at-risk drinkers. Alcohol 2006;40:41-9.

8. Rospenda KM, Minich LM, Milner LA, Richman JA. Caregiver burden and alcohol use in a community sample. J Addict Dis 2010;29:314-24.

9. Vaishnavi R, Karthik MS, Balakrishnan R, Sathianathan R. Caregiver burden in alcohol dependence syndrome. J Addiction 2017;5.

10. Mattoo SK, Nebhinani N, Kumar BA, Basu D, Kulhara P. Family burden with substance dependence: a study from India. Indian J Med Res 2013;137(4):704-11.

11. Sreekumar S, Subhalakshmi TP, Varghese PJ. Factors associated with resilience in wives of individuals with alcohol dependence syndrome. Indian J Psychiatry 2016;58(3):307-10.

12. First MB, Williams JBW, Karg RS, Spitzer RL: Structured Clinical Interview for DSM-5 Disorders, Clinician Version [SCID-5-CV]. Arlington, VA, American Psychiatric Association, 2015.

13. Beck, A.T., Ward, C. H., Mendelson, M., Mock, J., \& Erbaugh, J. [1961] An inventory for measuring depression. Archives of General Psychiatry, 4, 561-571.

14. Beck AT, Steer RA, Garbin MG. Psychometric properties of the Beck Depression Inventory: Twenty-five years of evaluation. Clin Psychol Rev 1998;8(1):77-100.

15. Stockwell TI, Sitharthan T, McGrath DA, Lang E. The measurement of alcohol dependence and impaired control in community samples. Addiction 1994;89(2):167-84. 
16. Brown S, Biegel DE, Tracy EM. Likelihood of asking for help in caregivers of women with substance use or co-occurring substance use and mental disorders. Care Manage J 2011;12(3):94-100.

17. Wei Y, McGrath PJ, Hayden J, Kutcher S. Mental health literacy measures evaluating knowledge, attitudes and help-seeking: a scoping review; BMC Psychiatry 2015;15:291.

18. Mattoo SK, Ghosh A, Basu A. Substance use and family burden: A narrative review. Indian J Soc Psychiatry 2019;35(3):158-63.

19. Shekhawat BS, Jain S, Solanki HK. Caregiver burden on wives of substance-dependent husbands and its correlates at a Tertiary Care Centre in Northern India. Indian J Pub Health 2017;61(4):274-7.

20. Hoertel N, Crochard A, Limosin F, Rouillon F. Excessive alcohol consumption: what is the burden on natural caregivers?. L'encephale 2014;40:S1-10.

$* * * * * * * * * * * * * * * * * * * * * * * * * * * * * * * * * * * *$

Acknowledgements - Nil

Conflict of Interest - Nil

Funding - Nil 